\title{
Die chemische Abscheidung der bei der Spaltung des Urans entstehenden Elemente und Atomarten I.
}

\author{
Von Otto Hahn, Fritz Strassmann und Walter Seelmann-Eggebert
}

(Z. Naturforschg. 1, 545-556 [1946]; eingegangen am 5. August 1946)

Aus dem Kaiser-Wilhelm-Institut für Chemie, Tailfingen

\begin{abstract}
Es wird die chemisch-analytische Abtrennung der bei der Uranspaltung auftretenden Elemente beschrieben, unter besonderer Berücksichtigung der durch die Radioaktivität gegebenen Notwendigkeiten.

Im 1. Abschnitt wird eine Vorschrift über die Abtrennung aller vorkommenden analytischen Gruppen gegeben. Da nun aber die Abtrennung sämtlicher Spaltelemente aus dem Uran nebeneinander nicht ganz einfach und recht zeitraubend ist, wird im 2. Abschnitt neben der Abtrennung der Halogene, Edelgase, Alkalien und Erdalkalien aus den einzelnen Gruppen in der Hauptsache die direkte Abtrennung der einzelnen Elemente aus dem Uran beschrieben, besonders auch für den Fall, daß schnellstes Arbeiten notwendig ist.
\end{abstract}

Abschnitt 1, Isolierung sämtlicher bei der Spaltung entstehenden Elemente aus einer Probe bestrahlten Urans

\section{$\mathrm{D}$} as mit Neutronen bestrahlte, die Spaltprodukte enthaltende Uran (am besten Uranylnitrat) wird in der bei den Edelgasen später beschriebenen Weise in einer Waschflasche in Wasser gelöst und von den Edelgasen befreit. Liegt das Uran als Ammondiuranat vor, so löst man in kalter Salpetersäure.

2. Die Lösung wird in ein Becherglas gespült und für je $10 \mathrm{~g}$ Uran auf $100 \mathrm{bis} 200^{\circ} \mathrm{ccm}$ verdünnt. Dann wird so viel Essigsäure zugesetzt, daß die Lösung 2-3-proz. essigsauer wird, und schließlich wird in der Wärme mit Natriumacetat das Uran als Natriumuranylacetat ausgefällt. (Zink würde mitfallen.)

3. Das nur noch wenig Uran enthaltende essigsaure Filtrat wird mit einem oder mehreren Trägern für die Schwefelwasserstoffällung versetzt (etwa Ruthenium, Rhodium, Indium, Cadmium, Zinn, Antimon und Kupfer) und in der Kälte mit Schwefelwasserstoff gesättigt. Die Elemente Molybdän, Element 43, Ruthenium, Rhodium, Palladium, Silber, Cadmium, Indium, Zinn, Antimon, Tellur und Kupfer fallen als Sulfide aus. Ferner würden Selen und Arsen fallen, falls anwesend, Germanium, Gallium, Nickel und Reste von Zink in Lösung bleiben. Zusatz von Silber ist nicht zu empfehlen, da sonst Silberhalogenid fällt und aktive Brom- und Jod-Isotope mitgefällt würden. Nur in stark chloridhaltigen Lösungen, in denen sich Silberchlorid komplex wieder löst, kann Silber wägbar als Sulfid gefällt werden, ohne daß Brom und Jod als Halogenide in den Sulfidniederschlag gehen.

4. Im Filtrat wird der Schwefelwasserstoff vertrieben (evtl. durch Einleiten von Schwefeldioxyd, wodurch zwar Schwefel ausfällt, aber die Bildung von Sulfat verhindert wird), dann werden Kaliumbromid und Kaliumjodid zugesetzt und mit Silbernitrat gefällt. Es fallen Brom und Jod als Silberhalogenide aus.

5. Im Filtrat wird der Überschuß an Silber mit wenig verd. Salzsäure gefällt, oder aus stark chloridhaltigen Lösungen durch Schwefelwasserstoff als Silbersulfid. Im letzteren Falle wird der Schwefelwasserstoff vertrieben, die Lösung weitgehend eingeengt und mit Salzsäure abgeraucht, bis Chlor bzw. Nitrosylchloride nicht mehr entweichen. Der Rückstand wird mit möglichst wenig 20-30-proz. Salzsäure aufgenommen. Es bleibt die Hauptmenge des nach Entfernung des Nitrats 
und der Essigsäure entstandenen Natriumchlorids unlöslich zurück.

6. Das stark salzsaure Filtrat wird eingeengt, bis die Hauptmenge der Salzsäure verdampft ist, dann wird mit Wasser aufgenommen und nach Zusatz von einigen Milligramm Ferrichlorid und wägbaren Mengen von Barium und Strontium (oder Calcium) und Rubidium und Cäsium mit Ammoniak gefällt. Es fallen mit dem Eisenoxydhydrat die Reste Uran, Zirkon, Niob, die Seltenen Erden, das Element 93 und Uran $X$.

7. Zum Filtrat setzt man weiteres Barium- und Strontiumchlorid und fällt mit Ammoncarbonat bzw. mit Natriumcarbonat. Befürchtet man, daß im Laufe der Verarbeitung Sulfat entstanden ist, so daß sich beim Zusatz von Bariumchlorid unlösliches Bariumsulfat abscheidet, so fällt man Barium und Strontium unwägbar mit Calciumcarbonat als Träger. Es fallen Barium und Strontium als Carbonate.

8. Das Filtrat wird mit Salzsäure angesäuert und eingedampft. Durch vorsichtiges Glühen werden die Ammonsalze vertrieben. Der Rückstand wird mit einem Gemisch von 37-proz. Salzsäure und Alkohol 2:1 aufgenommen und verrieben. Die Reste von Natriumchlorid bleiben ungelöst zurück, Rubidium- und Cäsiumchlorid gehen in Lösung. Alkohol und Salzsäure werden verdampft. Der Salzrückstand wird mit schwach salzsaurem Wasser aufgenommen und nacheinander nach Zusatz von 2-3 mg Kupfer-Ionen, EisenIonen und Barium, Strontium mit Schwefelwasserstoff, Ammoniak und Natriumcarbonat gefällt. Es fallen evtl. vorhandene Reste der betreffenden Gruppen, die bei zu großer Fremdsalzkonzentration vorher in Lösung geblieben sein können. Das Filtrat wird eingedampft und von den Ammonsalzen befreit. Der Rückstand wird mit 25-proz. Salzsäure aufgenommen und mit Kieselwolframsäure gefällt. Es fällt Cäsium als Cäsiumsilicowolframat. Das Filtrat wird nach vollständiger Fällung des Cäsiums in der Hitze mit alkohol. Zinnchlorid-Lösung gefällt. Es fällt Rubidium als Rubidiumzinnchlorid.

\section{Abschnitt 2, Halogene, Edelgase,} Alkalien und Erdalkalien

Da bei der Spaltung des Urans immer gleichzeitig zwei Elemente auftreten, die sich in ihren Kernladungszahlen zu 92 ergänzen, und da die bisher nachgewiesenen Bruchstücke und ihre Umwandlungsprodukte die Elemente der Kernladung 35 bis 59 einschließen, so finden sich unter diesen Substanzen eine ganze Anzahl homologer, chemisch einander ähnlicher Elemente, wie Brom und Jod, Krypton und Xenon usw. Um Wiederholungen zu vermeiden, werden diese homologen Elemente gemeinsam besprochen.

\section{A. Die Halogene Brom und Jod}

Das Brom mit seiner Kernladung 35 ist bisher das niedrigste Element, das als Spaltprodukt des Urans nachgewiesen wurde. Dies schließt nicht aus, daß noch niedrigere Spaltprodukte als Muttersubstanzen der Bromisotope auftreten. Sollte dies der Fall sein, dann haben diese aber eine so kurze Halbwertszeit, daß wir sie aus diesen nicht gewinnen können. Beim ${ }_{53}$ Jod dagegen sind außer Isotopen, die keine oder nur eine sehr kurzlebige Muttersubstanz besitzen, auch solche bekannt, die aus ${ }_{52}$ Tellur oder indirekt sogar aus ${ }_{51}$ AntimonIsotopen längerer Halbwertszeit entstehen. Bei der Suche nach Jod-Isotopen und ihrer Abscheidung aus dem Uran ist dieser Umstand zu berücksichtigen. Man glaubt, das Jod quantitativ nach einer zuverlässigen Methode abgeschieden zu haben, und kann es plötzlich an anderer Stelle wiederfinden; es hat sich dann aus seiner Muttersubstanz Tellur nachgebildet. Beim Brom tritt dieser Fall nicht ein.

Für die Abscheidung der Halogene aus dem Uran und ihre Trennung voneinander stehen mehrere Wege zur Verfügung, die Destillation, die Ausfällung mit Silber-Ionen und die Ausschüttelung mit organischen Lösungsmitteln.

\section{a) Brom}

Handelt es sich um die Erfassung kurzlebiger Brom-Isotope und die Verarbeitung nicht zu großer Mengen bestrahlten Urans $(<50 \mathrm{~g})$, so wird man immer die Destillation anwenden. Das bestrahlte Uranat wird in siedender $15-25$-proz. Schwefelsäure gelöst und die Lösung in einen Kolben mit Gasableitungsrohr gegossen, in dem sich einige Milligramm Kaliumbromid befinden; dann wird die äquivalente Menge Kaliumbromat zugesetzt, der Kolben verschlossen und die Lösung destilliert. Man kann damit rechnen, daß bei dieser Arbeitsweise das gesamte durch Spaltung entstandene Brom erfaßt wird, unabhängig davon, in wel- 
cher Form es entstanden ist oder im Augenblick der Destillation vorliegt, weil sowohl Bromide wie auch Bromat und freies Brom als inaktive Träger in der Lösung vorhanden sind oder doch kurze Zeit vorhanden waren. Das Ableitungsrohr des Destillationskölbchens taucht in einen kleinen Erlenmeyer mit etwas Natriumbisulfitlösung, in der das Brom zu Bromid reduziert wird. Nach Beendigung der Destillation (wenn die deutlich sichtbare Hauptmenge Brom, $50 \mathrm{mg}$, übergegangen ist) säuert man den Inhalt des Erlenmeyers mit Salpetersäure an und fällt Silberbromid. Der Niederschlag wird auf einem gehärteten Filter abgesaugt und mit Wasser gewaschen. Bei Abwesenheit wägbarer Mengen von Jod werden die durch Spaltung entstandenen aktiven JodIsotope durch das freie Brom bis zum Jodat oxydiert, so daß eine Trennung Brom-Jod durch Destillation recht gut gelingt.

Sind erheblich größere Mengen Uran zu verarbeiten, so empfiehlt es sich, zunächst die Abscheidung aus dem Uran als Silberbromid und Silberjodid mit wägbarem Brom-Ion als Träger vorzunehmen. Man verwendet dazu natürlich unter keinen Umständen Uranylsalze, deren Anionen mit dem Silber-Ion einen schwer löslichen Niederschlag ergeben würden, also z. B. eine salzsaure Lösung von Natriumdiuranat, sondern stets Nitrat- oder Acetatlösungen. Zwar sind Silberbromid und Silberjodid so viel schwerer löslich als Silberchlorid, daß sie sich unter allen Umständen mit dem ersten Anteil des Silberchlorids niederschlagen würden, doch tritt eine Niederschlagsbildung in diesen verhältnismäßig konzentrierten Chloridlösungen auf Zusatz von 20 bis $30 \mathrm{mg}$ Silber-Ion gar nicht ein, da sich das Silberchlorid genau wie in starker Salzsäure oder in Alkalichloridlösung sofort zum SilberchloridIon-Komplex löst. Man setzt also zur schwach salpetersauren Lösung des bestrahlten Urans $30-50 \mathrm{mg}$ Brom-Ion und fällt mit einem Überschuß an Silbernitrat das Silberbromid aus. Die Erfahrung zeigt, daß man mindestens einen erheblichen Teil der entstandenen Halogene mit Silber-Ion ausfällen kann. Befürchtet man aber etwa das Vorhandensein von Bromat und freiem Brom, so setzt man vor der Fällung 2-3 ccm Schweflige Säure zur Uranylsalzlösung, wodurch eine Reduktion der verschiedenen Oxydationsstufen zum Bromid erfolgt, ohne die Uranylsalzlösung $\mathrm{zu}$ beeinflussen.
Eine Trennung Bromid-Jodid läßt sich so aber nicht durchführen, da, wie bereits erwähnt, das schwerer lösliche Silberjodid sich mit dem Silberbromid gemeinsam abscheidet, gleichgültig, ob es in wägbarer Form vorliegt oder nicht. Die Fällung dient also nur als Anreicherungsverfahren. Der Niederschlag wird mit Zink und Schwefelsäure reduziert. $\mathrm{Zu}$ diesem $\mathrm{Zweck}$ wird der auf einem gehärteten Filter abgesaugte Niederschlag in ein Becherglas mit verd. Schwefelsäure gebracht und mit Zinkstaub. überschichtet. Nach einigen Minuten kann der Silber- und Zinkrückstand abfiltriert werden. Legt man besonderen Wert auf schnelles Arbeiten, so setzt man die Nutsche, auf der man den Niederschlag abgesaugt hat, auf eine saubere Saugflasche, bedeckt den Halogensilberniederschlag auf dem Filter mit einer dünnen Schicht Zinkstaub und saugt dann $30-50 \mathrm{ccm}$ verd. Schwefelsäure durch das Filter. Im silberfreien Filtrat befindet sich dann ein erheblicher Teil der Halogene. Die Lösung wird mit der äquivalenten Menge Kaliumbromat oder mit Kaliumpermanganat destilliert. Bei dieser Arbeitsweise (nur unwägbare Mengen von Jod) gelingt es wie oben, die Mitdestillation von Jod $\mathrm{zu}$ unterdrücken. Befürchtet man aber dennoch eine Verunreinigung durch das in viel größerer Intensität entstehende Jod und steht genügend Zeit zur Verfügung, so kann man auch zunächst das Jod abdestillieren und erst im Anschluß daran das Brom. Allerdings muß man dann sicher sein, daß das Jod vollständig abdestilliert worden ist.

Ein Ausschütteln des Broms mit Ather, Chloroform usw. ist nicht zu empfehlen, da die Löslichkeit des Broms in Wasser so groß ist, daß die Ausbeuten beim Ausschütteln sehr klein werden.

Bei Verwendung nicht zu großer Mengen von Uran und bei der eingangs erwähnten Destillation des Broms gelang es bei einiger Ưbung, das Brom $\sim 3,5$ Min. nach dem Ende der Bestrahlung zur Messung zu bringen.

\section{b) Jod}

Die Verarbeitung erfolgt ganz ähnlich wie beim Brom. Das bestrahlte Uranat wird in siedender 15-25-proz. Schwefelsäure gelöst, die Lösung mit Kaliumjodid versetzt (entspr. 30-50 mg Jod) und mit Natriumnitrit, Eisenchlorid oder Kaliumjodat destilliert. Unter diesen Umständen destilliert nur Jod. Das Destillat wird in Natriumbisulfit aufgefangen und nach dem Ansäuern mit 
Salpetersäure mit Silber-Ionen gefällt. Bei größeren Mengen Uran kann Jod gemeinsam mit Brom als Silberjodid gefällt und mit Schwefelsäure + Zink reduziert werden. Anschließend findet dann die Destillation statt. Bei schnellem Arbeiten gelang es, das Jod 3-5 Min. nach dem Ende der Bestrahlung zur Messung zu bringen.

Mit gutem Erfolg kann aber auch die Lösung in der Kälte zunächst mit äquivalenten Mengen Kaliumjodid und Kaliumjodat versetzt werden. Nach dem Ansäuern läßt sich dann nach einigen Minuten das Jod mit Chloroform oder Schwefelkohlenstoff ausschütteln. Das Ausschütteln wird einige Male wiederholt. Das Ex́traktionsmittel wird jedesmal im Scheidetrichter von der Uranlösung getrennt und am besten gleich in einen anderen Scheidetrichter abgezogen, in dem die vereinigten Anteile des Extraktionsmittels mit etwas wäßriger Bisulfitlösung geschüttelt werden. Dadurch werden das Jod und die unwägbaren Mengen Brom rasch in Jodid bzw. Bromid übergeführt und gehen vollständig in die wäßrige Phase über, die im Schütteltrichter leicht von dem nunmehr farblosen Extraktionsmittel getrennt werden kann. Die Jodidlösung wird in einen Destillationskolben gebracht, mit verd. Schwefelsäure angesäuert, um das überschüssige Bisulfit zu zerstören, durch Kochen von Schwefliger Säure befreit und mit Natriumnitrit destilliert. Es wird nur Jodid zu Jod oxydiert. Die Hauptmenge des Jods destilliert sehr rasch. Die Weiterbehandlung erfolgt wie oben angegeben.

\section{c) Brom und Jod}

Sollen aus Vergleichsgründen Brom und Jod aus ein und derselben Probe bestrahlten Urans abgetrennt und gemessen werden, so wird man den Arbeitsgang nach den Erfordernissen des Versuches wählen. Soll zuerst das Brom zur Messung gelangen, so wird die schwefelsaure Lösung des bestrahlten Urans mit äquivalenten Mengen Kaliumbromid und Kaliumbromat versetzt und destilliert. Das nur unwägbar vorhandene Jod wird durch das in Freiheit gesetzte Brom zu nicht flüchtigem Jodat oxydiert, wie bereits oben erwähnt wurde. Ist ausreichend Zeit vorhanden, dann setzt man zum Inhalt der Vorlage 10-20 mg Kaliumjodid als Träger für etwa mitgerissene kleine Mengen der aktiven Jod-Isotope, füllt ihn in einen Destillierkolben, säuert mit verd. Schwefelsäure an, verkocht den Hauptteil der Schwefligen Säure und treibt dann zunächst nach Zusatz von hinreichend Natriumnitrit das Jod über. Im Anschluß daran wird durch Kaliumpermanganat das Brom in Freiheit gesetzt und ebenfalls destilliert. Es ist nunmehr frei von Jod. Bei der nun vorzunehmenden Abscheidung des Jods aus der Uranlösung muß man berücksichtigen, daß beim schnellen Arbeiten die Abdestillation des Broms aus der Uranlösung nicht quantitativ war. Es können also noch Reste von Brom bei der Joddestillation übergehen, so daß noch eine nachträgliche Reinigung des Jods zu empfehlen ist. Man vermeidet diese Schwierigkeit, wenn man nach Unterbrechung der Bromdestillation etwa $50 \mathrm{mg}$ Kaliumjodid zur Uranlösung in den Destillierkolben fügt und einige Minuten wartet, und dann Natriumnitrit zusetzt. Das etwa noch in Lösung vorhandene freie Brom oder ein Überschuß an Bromat setzen dann das Jodid unter Bildung von Bromid $\mathrm{zu}$ freiem Jod um; das restliche Jodid wird durch das zugesetzte Natriumnitrit oxydiert. Eine Reinigung von Brom ist unter diesen Umständen nicht mehr erforderlich.

Soll dagegen zuerst das Jod zur Messung gelangen, so wird aus der schwefelsauren Lösung mit Kaliumjodid und Natriumnitrit zunächst das Jod übergetrieben, das zwar in reiner Form übergeht, zu dessen quantitativer Destillation aber in den meisten Fällen ebenfalls die dazu erforderliche Zeit nicht zur Verfügung stehen wird. Es bleiben also beim Unterbrechen der Destillation wägbare Mengen Jod in der Lösung und zum Teil auch im Gasraum. Ein nunmehr erfolgender $\mathrm{Zu}$ satz von Kaliumbromid und Kaliumbromat setzt zwar Brom in Freiheit, doch läßt sich nicht verhindern, daß ein Teil des noch vorhandenen Jods mit dem Brom überdestilliert und durch die Bisulfitlösung zu Jodid reduziert wird. Es muß also in diesem Falle unter allen Umständen mit dem Destillat noch eine Trennung Brom-Jod durchgeführt werden, und zwar um so mehr, als gerade das Jod gegenüber dem Brom einmal in durchschnittlich zehnmal größerer Intensität entsteht und zum anderen über Muttersubstanzen verfügt (Antimon - Tellur - Jod), die nach Maßgabe ihrer Halbwertszeiten zum Teil noch nach vielen Stunden und sogar Tagen eine Nachbildung von Jod verursachen. Die Bisulfitlösung, die zum Auffangen des Broms bestimmt war, wird also mit einer erheblichen Menge Jodid versetzt $(\sim 50 \mathrm{mg}$ Kaliumjodid) und, wie oben angegeben, mit 
Schwefelsäure und Natriumnitrit vom Jod befreit. Dann wird das Brom aus salpetersaurer Lösung mit Silber-Ion gefällt und wie oben weiter verarbeitet.

\section{d) Sekundäres Jod}

Der Umstand, daß vom Jod (im Gegensatz zum Brom) zum Teil ziemlich langlebige Muttersubstanzen existieren, bietet die Möglichkeit, das Jod bei einmaliger Destillation nicht nur frei von anderen Substanzen $\mathrm{zu}$ erhalten, sondern sogar einzelne Jod-Isotope oder doch Isotopengemische einfacherer Zusammensetzung, die der graphischen Analyse keine Schwierigkeiten bieten. So entsteht durch längere Bestrahlung des Urans eine größere Zahl von Tellur-Isotopen. Isoliert man mindestens 10-12 Stdn. nach dem Ende der Bestrahlung das Tellur entweder durch Fällung mit Schwefliger Säure oder auch einfach durch Gruppenfällung mit Schwefelwasserstoff, so bildet das dann vorhandene Gemisch von TellurIsotopen nur noch zwei Jod-Isotope von 2,4 Stdn. und 8 Tagen Halbwertszeit nach. In ähnlicher Weise können bei starker Bestrahlung durch Isolierung von reinem Antimon aus dem Uran die Muttersubstanzen von zwei Tellur- und in diesem Falle auch von zwei Jod-Ispotopen gewonnen werden, die Halbwertszeiten von 2,4 und 18,5 Stdn. besitzen. Fällt man das Tellur, vom Ende der Bestrahlung ab gerechnet, mindestens erst nach 12-13 Tagen aus, so liefert das Isotopengemisch, das dann vorliegt, nur noch ein Jod von 2,4 Stdn. Halbwertszeit nach.

Jod aus Tellur. Das bestrahlte Uran wird in Lösung gebracht und z. B. mit etwa $10 \mathrm{mg}$ Kupfer-Ionen versetzt. Das Kupfer wird mit Schwefelwasserstoff gefällt. Mit dem Sulfidniederschlag fällt auch das Tellur. Der Niederschlag wird in einigen Tropfen Salpetersäure gelöst, wobei das Tellur in Tellurige Säure $\mathrm{H}_{2} \mathrm{TeO}_{3}$ übergeht. Die Säure wird verdampft, der Rückstand mit verd. Schwefelsäure aufgenommen. Aus dieser Lösung kann nach dem Versetzen mit Kaliumjodid und Natriumnitrit Jod abdestilliert werden, wenn eine zur Nachbildung ausreichende Zeit vergangen ist. An Stelle der Sulfidfällung kann man auch $10-20 \mathrm{mg} \mathrm{H}_{2} \mathrm{TeO}_{3}$ zur Uranlösung setzen und das Tellur mit Schwefliger Säure ausfällen. Der Niederschlag wird in Salpetersäure gelöst, die überschüssige Säure wird verdampft und der Rückstand mit verd. Salzsäure oder Schwefelsäure aufgenommen. Das aus dem Tellur entstandene Jod wird dann, wie oben angegeben, abdestilliert.

Jod aus Antimon. Diese Abtrennung läßt sich mit Erfolg nur dann durchführen, wenn eine ausreichend starke Strahlenquelle zur Verfügung steht. Die in Frage kommenden Antimon-Isotope haben Halbwertszeiten von nur 5 und 10 Min., die daraus entstehenden Tellur-Isotope haben Halbwertszeiten von $77 \mathrm{Stdn}$. bzw. 60 Min. und gehen in Jod-Isotope von 2,4 und 18,5 Stdn. Halbwertszeit über. Das Antimon muß also so schnell wie möglich in reiner Form isoliert werden. Die Bestrahlungsdauer braucht höchstens 1 Stde. zu betragen, dann wird das bestrahlte Uranat in verd. Salzsäure gelöst. Aus der schwach sauren Lösung werden 10-20 mg Antimon als Antimonsulfid gefällt; der Niederschlag wird in Salzsäure gelöst und in die Lösung nach dem Vertreiben des Schwefelwasserstoffs in der unter Antimon zu beschreibenden Weise metallisches Zink gegeben (Gegenwart von wägbarem Tellur). Das sich entwickelnde Gemisch von Wasserstoff und Antimonwasserstoff wird in eine Silbernitratlösung eingeleitet. Der dabei entstehende Niederschlag wird unter Zusatz von Salpetersäure gelöst und die Lösung eingedampft. Der Salzrückstand wird mit verd. Schwefelsäure aufgenommen und die klare Lösung nach entsprechender Wartezeit nach Zusatz von etwas Salpetersäure mit 10-20 mg Kaliumjodid versetzt. Es fällt Silberjodid, das als solches gemessen wird. Entsteht beim Eindampfen des antimonhaltigen Silberniederschlages mit Salpetersäure ein schwerlöslicher Rückstand, so kann er leicht mit einigen Körnchen Weinsäure (Bildung leicht löslicher Antimontartrate) in Lösung gebracht werden.

\section{B. Die Edelgase Krypton und Xenon}

\section{a) Indirekter Nachweis}

Nachdem bei der Bestrahlung des Urans mit Neutronen das Auftreten von Barium, und zwar gleich in Gestalt mehrerer Isotope, nachgewiesen war, wurden sofort Versuche unternommen, Isotope des Edelgases Krypton nachzuweisen, weil bei der Entstehung des ${ }_{56}$ Bariums aus dem ${ }_{92}$ Uran das ${ }_{36}$ Krypton entstehen mußte. Durch eine Lö-. sung von Uranylnitrat wurde während der Bestrahlung ein Luftstrom geleitet, der nach der Uranlösung ein Wattefilter und darauf ein mit 
angesäuertem Wasser beschicktes Absorptionsgefäß passierte. In dem Wasser ließen sich unschwer bei den vorher zugegebenen Alkali- und Erdalkalisalzen Aktivitäten nachweisen, die also aus mit dem Luftstrom übergeführten künstlichen aktiven Edelgasen stammen mußten. Später wurde statt des Wassers Adsorptionskohle zum Auffangen der Edelgase bzw. ihrer Umwandlungsprodukte gewählt. Der Luftstrom wurde nacheinander durch einen Wattefilter, ein Trockenrohr und ein mit Alkohol-Kohlensäure tief gekühltes U-Rohr mit Adsorptionskohle geleitet. Die Kohle wurde nach Beendigung der Bestrahlung der Uranlösung mit warmer verd. Salzsäure nach Zugabe von Alkali- und Erdalkalisalzen extrahiert, und aus der Lösung wurden Rubidium, Cäsium, Strontium und Barium einzeln abgeschieden. Nach kurzen Vorversuchen wurden aktive Isotope aller dieser vier Elemente, außerdem auch noch Yttrium- und Lanthanisotope nachgewiesen. Damit war bewiesen, daß bei der Zerspaltung des Urans nicht nur ${ }_{56}$ Barium und ${ }_{36}$ Krypton $(56+36=92)$, sondern auch ${ }_{54}$ Xenon und ${ }_{38}$ Strontium $(54+38=92)$ entstehen.

Durch Anwendung verschiedener Kältebäder, nämlich.1. Eis-Kochsalz,2.Kohlendioxyd-Alkohol, 3. flüssige Luft, und bestimmter Strömungsgeschwindigkeiten des Luftstromes konnte sogar eine so gute Trennung von Krypton und Xenon erzielt werden, daß z. B. die mit Eis-Kochsalz gekühlte Kohle nur Xenon, Cäsium, Barium und Lanthan enthielt, die mit flüssiger Luft gekühlte Kohle dagegen nur Krypton, Rubidium, Strontium und Yttrium. In der mit Kohlensäure-Alkohol gekühlten Kohle fanden sich je nach der Strömungsgeschwindigkeit überwiegend Xenon, Cäsium, Barium und Lanthan neben den Spalt- bzw. Umwandlungsprodukten niedrigerer Ordnungszahl.

Für Edelgase, deren Halbwertszeiten mehr als einige Minuten betragen, läßt sich nach diesen indirekten Methoden angenähert auch eine ungefähre Bestimmung der Halbwertszeit durchführen. Man bestrahlt die Uranlösung in einer verschlossenen Waschflasche so lange, daß die Erreichung des Gleichgewichtes wahrscheinlich ist, oder aber man bestrahlt immer genau gleich lange. Nach dem Ende der Bestrahlung leitet man dann sofort oder in verschiedenen Zeitabständen durch die gleichmäßig bestrahlten Uranlösungen einen Luftstrom immer gleicher Strömungsgeschwindig- keit und erhält dann nach entsprechender Wartezeit für die Nachbildung der Umwandlungsprodukte aus der Kohle Aktivitäten (etwa von Cäsium oder Barium), deren Anfangsintensitäten entsprechend den Wartezeiten und der Halbwertszeit des Edelgases (etwa Xenon) abnehmen.

\section{b) Direkter Nachweis}

Weit zuverlässiger, vor allem auch für kurzlebige Edelgase, ist die direkte Messung der Edelgase selbst in besonderen, zu diesem Zweck konstruierten Zählrohren ${ }^{1}$. Bei dem einfachsten Modell wird um ein normales Zählrohr von $100 \mu$ Wandstärke ein starkwandiger Mantel unmittelbar befestigt, der durch zwei kleine Stutzen mit Glashähnen versehen ist. Mittels dieser kann der Mantel evakuiert und dann schnell mit dem zu untersuchenden Gas gefüllt werden; ebenso schnell läßt sich das Mantelgefäß auch wieder auspumpen, was $u$.U. für die Prüfung auf kurzlebige Umwandlungsprodukte der aktiven Edelgase notwendig ist.

Bei stärkeren Aktivitäten hat sich folgende Anordnung sehr bewährt.

Das Mantelgefäß hat einen größeren inneren Durchmesser als der äußere des Zählrohrs und ist nicht mit dem Zählrohr verbunden. Wird es konzentrisch über das senkrecht stehende Zählrohr gestellt, dann bleibt ein gewisser Zwischenraum, in den adsorbierende Metallfolien eingebracht werden können. Mit Hilfe dieser Anordnung kann also außer der Halbwertszeit auch die Durchdringungsfähigkeit der Strahlung des Gases bestimmt werden. Der Nachteil ist der, daß wegen des größeren Abstandes der Kammer vom Zählrohr größere Aktivitäten notwendig sind und nur ziemlich durchdringende Strahlen gemessen werden können. Denn die Strahlung muß ja außer der Zählrohrwand von $100 \mu$ Dicke noch die innere Wand der Gaskammer durchdringen. Bei ihr wird man aus Stabilitäts- und Dichtigkeitsgründen kaum unter $150 \mu$ inneren Wanddurchmesser gehen können, so daß die Strahlung nicht wie vorher $100 \mu$, sondern jetzt $100+150 \mu$ Aluminium zu durchsetzen hat. Durch die Möglichkeit des Einbringens verschiedener Absorptionsfolien hat man dafür den Vorteil, daß man nicht nur die Härte der Strahlung einzelner Isotope bestimmen, sondern $u$. U. auch neue Isotope

1 W. S e e l m a n n - E g g e b e r t, Naturwiss. 28, 451 [1940]. 
neben schon bekannten auffinden kann, die sonst vielleicht übersehen worden wären, wie dies z. B. für den Fall eines Molybdän-Isotops von 12 Min. Halbwertszeit früher beschrieben wurde ${ }^{2}$.

Die Einbringung der zu untersuchenden Gase in die genannten Mantelrohre geschieht etwa folgendermaßen: Durch die in einer verschlossenen Gaswaschflasche bestrahlte Uranlösung wird ein Wasserstoffstrom hindurchgeschickt, der die in der Lösung während der Bestrahlung mit Neutronen gebildeten Edelgasatome auswäscht. Dieser Wasserstoffstrom wird nach dem Trocknen und nach Absorption der mitgerissenen Säuredämpfe durch ein mit Aktivkohle gefülltes, tiefgekühltes U-Rohr geleitet. Je nach der Temperatur der Kohle werden dann praktisch nur Xenon oder nur Krypton oder beide adsorbiert (siehe oben).

Bei Bestrahlung fester Salze tritt nur eine unwesentliche Anderung in der Arbeitsweise ein; man arbeitet wie vorher, nur wird vor dem Ausspülen mit Wasserstoff in die Gaswaschflasche, in der sich das bestrahlte Salz befindet, Lösungsmittel hinzugegeben. Man muß nur darauf achten, daß keine Gasverluste beim Lösen der Salze entstehen. Das durch das Lösungsmittel verdrängte Gas wird sofort durch das Adsorptions-U-Rohr geleitet. Bei Einhaltung dieser Vorsichtsmaßnahme kann genau wie vorher verlustfrei gearbeitet werden.

Das Auswaschen aus größeren Volumen oder sehr konzentrierten Lösungen dauert in der Kälte lange; man erwärmt daher besser. Schneller, d. h. in etwa 10 Sek., werden die Gase zu 98\% aus ungefähr $30 \mathrm{ccm}$ und etwa $70^{\circ}$ heißer Lösung ausgewaschen, wenn man das Waschgas aus einer Filterplatte in kleinen Bläschen austreten läßt. Dann genügt schon der Durchgang von $1 / 2 l$ Wasserstoff, um die aktiven Edelgase vollständig auszuwaschen. Sind nun die Gase, die gemessen werden sollen, an der gekühlten Aktivkohle adsorbiert, so wird das U-Rohr mit der evakuierten Kammer verbunden. Die Hähne gegen die Außenluft bleiben verschlossen, das U-Rohr wird in kochendes Wasser getaucht, wobei die adsorbierten Gase desorbiert werden. Bei Öffnen des zweiten Hahnes am U-Rohr gegen die Atmosphäre werden nun die aktiven Gas-Isotope durch die einströmende Luft in die Meßkammer getrieben.

${ }^{2} \mathrm{Hahn}$, Straßmann u. Götte, Abh. preuß. Akad. Wiss., Math.-naturwiss. Kl., Jhrg. 1942, Nr. 3, S. 26 .
Das Adsorbieren und Desorbieren der Edelgase Xenon und Krypton ist bei entsprechender Temperatur der, Kohle quantitativ. Man kann also diese beiden Edelgase aus dem Meßraum wieder an aktiver Kohle sammeln und später wieder zur Messung bringen, ohne daß Verluste auftreten. Bis zu einer Temperatur von etwa $-100^{\circ}$ bei Verwendung von grobkörniger Aktivkohle wird so wenig Wasserstoff adsorbiert, daß man mit einem Volumenverhältnis von Meßraum zu U-Rohr wie $6: 1$ quantitativ arbeitet, weil die nach Desorption der Edelgase und des Wasserstoffs nachströmende Luft alle aktiven Gasatome in die Meßkammer treibt. Bei tieferer Temperatur muß man bei diesem Volumenverhältnis und der gleichen Kohlenmenge mit Verlusten rechnen. Bei Verwendung der halben Kohlenmenge, Kühlung mit flüssiger Luft und unter sonst gleichen Bedingungen sind keine wesentlichen Verluste aufgetreten, wenn vor Beginn des Auswaschens die ganze Apparatur von Luft befreit worden war.

Bei der Messung von aktiven Gasen werden natürlich auch die evtl. aktiven Folgekörper, also Isotope von Alkalien, Erdalkalien oder Erden im Betrage ihrer Nachbildung mitgemessen. Dieser „aktive Niederschlag“ kann durch negatives Aufladen der Zählrohrhülle oder der äußeren Gaskammerwand im Verhältnis zur Aktivität der Gase verstärkt oder geschwächt gemessen werden.

Der aktive Niederschlag haftet so fest am Metall, daß er auch durch einen kräftigen Luftstrom nicht entfernt werden kann. Es ist deshalb möglich, auch sehr kurzlebige Folgekörper von aktiven Edelgasen nach Absaugen des Gases nachzuweisen, da dieses Aussaugen leicht im Bruchteil einer Sekunde vollständig mit Hilfe einer Wasserstrahlpumpe geschehen kann.

Die unmittelbare Messung der Halbwertszeiten der Edelgase ist wegen der aus ihnen entstehenden komplexen Umwandlungsprodukte nicht leicht durchzuführen, besonders wenn Edelgasgemische vorliegen. Die Eigenschaft der festen Folgeprodukte, sich zu fast $100 \%$ an negativ geladenen Platten niederzuschlagen, kann aber dazu benutzt werden, die Halbwertszeit der Gase auf Grund der Intensitätsabnahme der Folgeprodukte eines Gasgemisches zu bestimmen. Zu diesem Zwecke werden die aktiven Gase an Kohle adsorbiert und dann in ein Gefäß gebracht, in dem sich ein negativ geladenes Blech befindet. Auf diesem Blech schlagen sich nun die Folgeprodukte der Gase 
nieder. Nach einer bestimmten Zeit wird das Gas wieder aus dem Gefäß an gekühlter Kohle vollständig gesammelt, das Blech ausgewechselt und das Gas wieder hineingebracht. Der aktive Niederschlag wird mit verd. Säure vom Blech gelöst und nach chemischer Trennung das gewünschte Element abgeschieden und gemessen. In gleicher Weise wird mehrmals verfahren. Aus der Aktivität des Folgeproduktes, immer zu gleicher Zeit nach der Abtrennung vom Gas, wird nun die Halbwertszeit der gasförmigen Muttersubstanz bestimmt. Bei einer Blechoberfläche von etwa $5 \mathrm{qcm}$, einer Spannungsdifferenz von etwa $750 \mathrm{~V}$ und einem Volumen der Glasflasche von $150 \mathrm{ccm}$ wurden im Durchschnitt bei vielen übereinstimmenden Versuchen etwa 98\% des aktiven Folgekörpers auf dem geladenen Blech niedergeschlagen, während nur etwa $2 \%$ von den Glaswänden abgelöst werden konnten. Hier kann also die Halbwertszeit des Gases an ein und demselben Uranpräparat bestimmt werden, während in dem weiter oben beschriebenen Fall für jeden Meßpunkt eine neue Bestrahlung notwendig war.

\section{c) Indirekte Bestimmung von Halogenen durch die aus ihnen entstehenden Edelgase}

In ähnlicher Weise lassen sich durch wiederholtes Abtrennen der Folgekörper von der Muttersubstanz nach einer immer gleich bleibenden Wartezeit die Halbwertszeiten gewisser, sich in aktive Edelgase umwandelnder Halogenisotope genau bestimmen, welche sich wegen der Anwesenheit vieler aktiver Halogenisotope und deren Folgekörper durch Analyse der direkt gemessenen Abfallskurven nicht oder nur sehr annähernd bestimmen lassen. Auch bei Volumenänderungen der die Halogene enthaltenden Lösung, bei Veränderung der Temperatur, der Strömungsgeschwindigkeit und der zum Auswaschen benutzten Gasmenge ist die erzielte Genauigkeit gut.

Es sollen nun noch einige Angaben gemacht werden über die Dauer der oben beschriebenen Verfahren, da die Zeit bei kurzlebigen Substanzen oft von ausschlaggebender Bedeutung ist.

Es gelang, etwa 50\% des bei der Spaltung des Urans gebildeten Edelgasgemisches etwa 45 Sek. nach Ende der Bestrahlung zur Messung zu bringen, durch verschieden tief gekühlte Kohle (siehe oben) zu etwa $98 \%$ reines Xenon und Krypton in einer Ausbeute von etwa $5-\mathbf{1 0 \%}$ in ungefähr 3 Minuten. Handelt es sich um die Messung solcher
Xenon- und Krypton-Isotope, die aus primär entstandenen Jod- und Brom-Isotopen entstehen, und will man nur diese sekundären Edelgase untersuchen, dann muß das Jod oder das Brom vorher vom Uran abgetrennt werden. Dies kann nach der bei den Halogenen beschriebenen Destillationsmethode so schnell geschehen, daß das aus diesem Jod nachgebildete Krypton nach etwa 3,5 Min., berechnet vom Ende der Bestrahlung ab, zur Messung kommen kann. Die Ausbeute betrug bei diesem schnellen Arbeiten bis zu 70\%.

Bei quantitativer Gewinnung der Edelgase aus einer Halogenlösung, wie sie zur Bestimmung der Halbwertszeiten der Halogenmuttersubstanzen notwendig ist, kann mit der Messung etwa 3 Min. nach Beginn des Auswaschens begonnen werden.

Handelt es sich um den Nachweis von Edelgasen, deren Strahlen derartig absorbierbar sind, daß sie im 100- $\mu$-Zähler praktisch nicht mehr zur Messung gelangen, so kann man das Gas unmittelbar in das Zählrohr einführen, in einer Apparatur, wie sie von $\mathrm{Hahn}$ und $\mathrm{S}$ tr a $ß \mathrm{~m}$ a n n zur Untersuchung sehr stark absorbierbarer Strahlen fester Spaltprodukte mit Erfolg verwendet wurde ${ }^{3}$. Die Messung in einer solchen Zählanordnung erfordert allerdings eine wesentlich längere Zeit zur Isolierung und zum Einbringen in das Zählrohr. Dieser Zeitverlust ist aber dann zu ertragen, wenn die nur auf diese Weise zu messenden Edelgas-Isotope eine relativ große Halb. wertszeit besitzen, was bei Isotopen mit stark absorbierbarer Strahlung anzunehmen ist, wenn es sich nicht um Isomere handelt.

\section{Die Alkalimetalle Rubidium und Cäsium}

a) Abtrennung von Rubidium- und Cäsium-Isotopen, die aus Krypton und Xenon entstehen

Bei den Alkali-Isotopen, die aus Edelgasen entstehen, verwendet man zweckmäßig die leichte räumliche Trennung der gasförmigen Muttersubstanzen von bestrahltem Uran zu ihrer Gewinnung. Diese räumliche Trennung geschieht entweder, wie schon oben beschrieben wurde, durch Hindurchleiten eines Luftstromes durch das Uran während (oder u. U. nach) dessen Bestrahlung und darauffolgende Adsorption der Edelgase an gekühlte Kohle oder aber durch Sammeln der aktiven Folgeprodukte, des ,aktiven Niederschlages“ der Edelgase aus einem „hochema-

3 F. S t r a $B$ m a n n u. O. H a h n, Naturwiss. 30, 257 [1942]. 
nierenden Uranpräparat" ${ }^{\text {"4 }}$ an einer negativ aufgeladenen Metallplatte. In einer früheren Mitteilung ${ }^{5}$ wurden die Bedingungen diskutiert, unter denen diese Verwendung der „Emanierfähigkeit“ von Uranpräparaten eindeutige Ergebnisse liefert. Sie seien hier noch einmal kurz zusammengefaßt:

1. Das bestrahlte Uran muß in einer solchen Form vorliegen, daß auch sehr kurzlebiges Xenon oder Krypton aus der Uranverbindung in erheblicher Menge herausdiffundiert. Es muß also außerordentlich oberflächenreich sein. Die Ausfällung des Ammondiuranats geschieht deshalb nicht in der üblichen Weise, sondern durch schlagartiges Ausfällen in der Kälte; daß der dabei entstehende feine Niederschlag sich nur langsam filtrieren läßt, muß man in Kauf nehmen.

2. Auf der den ,,aktiven Niederschlag“" sammelnden Platte dürfen sich nicht auch die bei der Uranspaltung entstehenden Rückstoßteilchen befinden. Denn man will ja sicher sein, daß man nur die Umwandlungsprodukte der Edelgase auf der Platte hat. Daß auch diese Bedingung erfüllt ist, ließ sich dadurch feststellen, daß sich bei unseren Strahlenquellen keinerlei der mit Schwefelwasserstoff ausfällbaren Spaltprodukte bei der Verarbeitung des aktiven Niederschlages auf der Platte nachweisen ließen (bei Verwendung sehr starker Strahlenquellen und dünner Schichten Uran trifft dies natürlich nicht mehr ganz zu).

3. Das Metallblech, auf dem der aktive Niederschlag gesammelt wird, soll selbst nicht oder möglichst wenig durch die Neutronen aktiviert werden. Zumindest aber muß sich diese Aktivität von den Spaltprodukten leicht abtrennen lassen. Dies ist für die verschiedensten Metalle der Fall. Bei kurzer Bestrahlung wurde Cadmium gewählt, bei längerer Blei, natürlich kann man auch Kupfer, Edelmetalle und dergl. verwenden.

Ist der aktive Niederschlag an Blei niedergeschlagen, dann geschieht die Ablösung mit verd. Salzsäure, aus der etwa nach Zugabe der später abzuscheidenden Alkali- und Erdalkalimetalle die geringen Mengen aufgelösten Bleis leicht

4 Dieser nicht ganz korrekte Ausdruck soll besagen, daß das Uran in einer Form vorliegt, daß die in ihm entstehenden Edelgase $\mathrm{zu}$ einem hohen Prozentsatz nach außen diffundieren, ähnlich wie man von ,hochemanierenden" Radium- oder Thorpräparaten spricht, wenn das Radon oder Thoron aus ihnen herausdiffundieren kann.

5 O. H a h n u. F. St r a $B$ m a n n, Naturwiss. 28, 55 ['1940]. mit Schwefelwasserstoff entfernt werden können.

Die Fällung der Alkalimetalle kann als Perchlorat, Chloroplatinat, Chlorostannat usw. erfolgen, doch wird man meist das letztere vorziehen, da die vorliegende Lösung schon mineralsauer ist und das Chlorostannat aus 25-proz. $\mathrm{HCl}$ gefällt wird. Die Fällung erfolgt in der Siedehitze rasch, ist grob kristallin, kann auf einem gehärteten Filter abgesaugt und mit 25proz. Salzsäure und Alkohol gewaschen werden. Wie alle oben angegebenen Fällungsformen ist aber das Chlorostannat nicht spezifisch, sondern fällt sowohl Rubidium wie auch Cäsium, und da beide Verbindungen Mischkristalle bilden, spielt es auch keine Rolle, ob beide Elemente in wägbarer Menge vorhanden sind oder nicht. Wenn also nicht durch die Wahl der Kältebäder und Strömungsgeschwindigkeiten für eine Trennung der Edelgase gesorgt wurde, mißt man im Chlorostannat-Niederschlag die Summe der entstandenen Alkalien. In einem zweiten entsprechend bestrahlten Versuch isoliert man dann das Cäsium allein durch Fällung mit Kieselwolframsäure aus 25-proz. Salzsäure in der Kälte. Durch graphische Analyse beider Kurven erhält man dann einen Überblick über die Zahl und die ungefähren Halbwertszeiten der Alkali-Isotope.

Unter entsprechender Berücksichtigung der Löslichkeiten der einander entsprechenden Rubidium- und Cäsiumsalze läßt sich die Trennung aber auch aus ein und derselben Lösung ausführen. Man extrahiert die Kohle oder behandelt das Bleiblech mit ca. $10 \mathrm{ccm}$ 25-proz. Salzsäure, die etwa 10-20 mg Cäsium und höchstens $1 \mathrm{mg}$ Rubidium enthält, wäscht mit 5-10 ccm kalter 25-proz. Salzsäure nach und fällt in der Kälte mit einer Lösung von ca. $0,1 \mathrm{~g}$ Silicowolframsäure in 1-2 ccm Wasser. Der Zusatz von Rubidium ist zu empfehlen, um eine Adsorption der gewichtslosen Mengen an dem außerordentlich oberflächenreichen Niederschlag zu verhindern. Ein größerer Gehalt an Rubidium ist $\mathrm{zu}$ vermeiden, um eine Mitfällung des ziemlich schwer löslichen Rubidiumsilicowolframats zu unterbinden.

Die Fällung des Cäsiums ist erst nach mehrstündigem Stehenlassen quantitativ. Daher entfernt man bei Zeitmangel den Rest des noch in Lösung befindlichen aktiven und inaktiven Cäsiums, ohne zu filtrieren, durch mehrmalige $\mathrm{Zu}$ gabe von neuen Mengen von Cäsium (je 10 bis $20 \mathrm{mg}$ ), und auch von Kieselwolframsäure (die 
kleine Menge von Rubidium zur Verhinderung der Adsorption genügt vom ersten Versuch her). Die Niederschläge sind außerordentlich feinkörnig und müssen auf Membranfiltern abgesaugt werden. Der erste Niederschlag wird mit 25-proz. Salzsäure und anschließend mit Äther gewaschen. Das Filtrat der mehrfachen Cäsiumfällungen wird zum Sieden erhitzt, mit $20 \mathrm{mg}$ Rubidium und mit ca. $1 \mathrm{ccm}$ einer 40-proz. alkoholischen Lösung von Zinntetrachlorid gefällt. Der Niederschlag wird auf einem gehärteten Filter abgesaugt, mit 25-proz. Salzsäure und dann mit Alkohol gewaschen. Die Zugabe von Strontium, Barium, Yttrium, Lanthan, Cer usw. scheint bei der Fällung des Silicowolframats zur Vermeidung der Adsorption gewichtsloser Mengen nicht unbedingt erforderlich zu sein, da bisher nie eine entsprechende Störung beobachtet wurde. Will man aber aus Sicherheitsgründen auf die Anwesenheit der genannten Metalle nicht verzichten, so müssen die Mengen so klein gehalten werden, daß z. B. bei Barium in der 25-proz. Salzsäure keine Fällung von $\mathrm{BaCl}_{2}$ - $2 \mathrm{H}_{2} \mathrm{O}$ erfolgt.

Die Einzelfällung kann bei Verwendung möglichst kleiner Fällungsvolumina so schnell durchgeführt werden, daß man das Cäsiumsilicow olframat etwa 1,2 bis 1,4 Min. und das Rubidiumzinnchlorid etwa 1,4 bis 1,6 Min. nach Ende der Bestrahlung zur Messung bringen konnte. Sollen beide Aktivitäten aus einem Versuch bestimmt werden, ist der Zeitbedarf bis zur Messung des Rubidiums natürlich erheblich größer.

\section{b) Fällung und Trennung aus Lösungen von Uranylsalzen}

Das Verfahren der Abscheidung aus der Uranlösung selbst ist naturgemäß viel zeitraubender und eignet sich daher nur zur Abtrennung längerlebiger Rubidium- und Cäsium-Isotope. Da bei der zur Verarbeitung sonst so bequemen Verwendung von Ammoniumpyrouranat stets erhebliche Mengen von Ammonsalzen in der Lösung sind, die die Reinigung und Abscheidung der Rubidiumund Cäsiumniederschläge erschweren, geht man in diesem Falle vorteilhaft vom Uranylnitrat aus. Das bestrahlte Salz wird in einem Schütteltrichter mit einer ausreichenden Menge Ather (auf je $10 \mathrm{~g}$ Uranylnitrat etwa $100 \mathrm{ccm}$ Ather) gelöst. Die dabei entstehende wässerige Phase von Uranylnitrat enthält praktisch alle Spaltprodukte neben wenig Uran und wird in ein Becherglas mit heißem Was- ser abgezogen. Durch nochmaliges Schütteln mit einer geringen Menge Wasser kann man die Ausbeute an Spaltprodukten verbessern, erhöht allerdings dabei auch beträchtlich die später noch abzutrennende Uranmenge. Immerhin kann man auf diese Weise in wenigen Minuten etwa 90\% des Urans von den Spaltprodukten abtrennen. Zur Entfernung der immer noch beträchtlichen Uranmenge kann man zwei Wege einschlagen.

\section{c) Fällung des Urans als Natriumpyrouranat}

Man wählt das Fällungsvolumen so, daß auf etwa $10 \mathrm{~g}$ Uranylnitrat 400-600 cem Wasser vorhanden sind, erhitzt zum Sieden, setzt $20 \mathrm{mg}$ Cäsium und 1-2 mg Rubidium zu und fällt das Uran mit ca. 2-n. Natronlauge. Der Zusatz erfolgt am besten aus der Bürette unter kräftigem Umrühren zunächst schnell, bis eine eben bleibende Trübung auftritt, die sich meist nach wenigen Minuten ohne weiteren Zusatz von Natronlauge verstärkt, dann tropfenweise, bis die Hauptmenge des Urans gefällt ist. Zum Schluß gibt man einen geringen UUberschuß an Natronlauge hinzu. Man beobachtet dabei meist eine merkliche Farbvertiefung des. Niederschlags, der sich bei vollständiger Fällung sofort als schweres Pulver absetzt und durch ein gehärtetes Filter abgesaugt werden kann. Bei Verwendung carbonatfreier Natronlauge istdie Fällung des Urans quantitativ. Da aber im Laufe der weiteren Verarbeitung $u$. a. doch noch eine weitere Hydroxyd-Fällung ausgeführt werden muß, schadet auch ein geringer Carbonatgehalt der Natronlauge, der etwas Uran auflöst, nicht. Erfolgt die Fällung zu schnell oder aus zu konzentrierter oder zu verdünnter Lösung, so wird der Niederschlag äußerst oberflächenreich, läßt sich nur langsam durch Membranfilter absaugen und adsorbiert die Alkalien zu erheblichem Prozentsatz.

Das von Uran, Uran X und auch einer Anzahl von Spaltprodukten befreite, das Cäsium und $\mathrm{Ru}$ bidium enthaltende Filtrat. wird eingedampft und zur Entfernung der Salpetersäure schließlich im bedeckten Becherglas einige Male mit konz. Salzsäure abgeraucht. Nach der Zerstörung des Nitrats wird der trockene Rückstand sehr sorgfältig nochmals mit 10-20 cem eines Gemisches von konz. Salzsäure und Alkohol (2:1) verrieben und filtriert. Hierdurch wird ein großer Teil des störenden Chlornatriums entfernt, ohne daß das Rubidium oder Cäsium mitentfernt werden. (Einwand- 
freier ist es, den Trockenrückstand in der 4-fachen Menge Wasser zu lösen und mit dem 2,5-fachen Volumen des Salzsäure-Alkohol-Gemisches das Natrium zu fällen, nach 2 Stdn. zu filtrieren, das Filtrat zu verdampfen und die Operation zu wiederholen, bis beim Zusatz von Salzsäure-Alkohol nur noch ein geringer Niederschlag erscheint. Das Filtrat wird verdampft, mit schwach saurem Wasser aufgenommen und muß nun von den außer dem Rubidium und Cäsium noch vorhandenen anderen Spaltprodukten befreit werden. Die noch vorhandenen Elemente der Schwefelwasserstoffgruppe wie Molybdän, Element 43, Palladium, Silber, Cadmium usw. werden durch wiederholte Fällung mit Schwefelwasserstoff und Wismut oder Kupfer als Träger entfernt. Abweichend vom normalen Gang der Analyse werden anschließend nach Zusatz von je 20-50 mg Barium- und Strontiumsalz die Erdalkalien durch Zusatz von Soda gefällt, nachdem vorher der Schwefelwasserstoff vertrieben und die Säure durch etwas Natronlauge neutralisiert worden war.

Die Fällung erfolgt in diesem Falle vor der Fällung der Erden, des Zirkons und der u.U. noch vorhandenen Reste von Uran und Uran X, da ja aus den Erdalkalien dauernd Erden nachgeliefert werden (die ursprünglich entstandenen Erden sowie Zirkon und Niob sind mit der Fällung des Urans entfernt worden). Das Filtrat wird schwach angesäuert, von Kohlensäure durch kurzes Kochen befreit und noch mit 2-5 mg Eisenchlorid versetzt als Träger für die nachgebildeten Erden und die Reste von Uran und Uran X usw. Dann wird mit kohlensäurefreiem Ammoniak gefällt. Das Filtrat wird eingedampft. Bei vorsichtigem Arbeiten ist der Gehalt an Natrium- und Ammoniumsalzen so gering, daß mit dem Rückstand ohne weiteres nach der oben beschriebenen Methode die Fällung von Cäsium und Rubidium nacheinander durchgeführt werden kann. Eine gemeinsame Fällung als Chloroplatinat kommt hier nicht in Frage, da sich im Filtrat noch die aktiven Halogene befinden, die ebenfalls schwerlösliche Platin-Halogenkomplexe liefern.

d) Fällung des Urans als Natriumuranylacetat $\left(\mathrm{Na} \cdot \mathrm{UO}_{2} \cdot \mathrm{CH}_{3} \cdot \mathrm{COO}\right)$ und als Oxychinolat

Für manche Zwecke (etwa für die Erfassung von Cadmium, Indium usw.) ist es vorteilhafter, die Fällung des Urans in saurer Lösung vorzunehmen, und zwar speziell in essigsaurer Lösung.
Die aus dem Schütteltrichter abgezogene wäßrige Uranylnitratlösung läßt man in ein Becherglas mit heißem, essigsäurehaltigem Wasser fließen, so daß auf etwa $10 \mathrm{~g}$ Uranylnitrat ca. 100-150 ccm vorhanden sind, Zusatz von Rubidium oder Cäsium ist nicht erforderlich, da Adsorption an dem grobkristallinen Niederschlag oder Mischkristallbildung mit dem Natriumuranylacetat nicht erfolgt. Dann wird in die heiße Lösung festes Natriumacetat eingerührt, zunächst in größeren Anteilen, vom Auftreten eines bleibenden Niederschlags ab in kleinen Anteilen. Der grobkristalline Niederschlag setzt sich sofort ab, so daß leicht geprüft werden kann, ob bei weiterem Acetatzusatz sich noch neuer Niederschlag bildet. Der Niederschlag wird auf einem gehärteten Filter abgesaugt, das nur noch sehr wenig Uran enthaltende Filtrat eingedampft und dann'zunächst auf Schwermetalle der Schwefelwasserstoffgruppe verarbeitet oder mit schwach essigsaurem Wasser aufgenommen und durch Fällung mit 8-Oxychinolin ( siehe Berg „Das Oxin“) von den Resten des Urans befreit. Eine Fällung mit Ammoniak ist hier nicht $\mathrm{zu}$ empfehlen, da aus stark acetathaltigen Lösungen das Uranat sehr oberflächenreich fällt. Das Filtrat aus dem Oxychinolin-Niederschlag wird eingedampft, wobei man es dauernd schwach ammoniakalisch hält und vorteilhaft einen schwachen Luftstrom zur Entfernung des Chinolins hindurchtreibt. Nach dem völligen Vertreiben des Oxychinolins entfernt man die übrigen Spaltprodukte durch aufeinanderfolgende Schwefelwasserstoff-, Natriumcarbonat- und Ammoniakfällungen, in der unter a) beschriebenen Weise. Es ist hier aber zu beachten, daß bei der Natriumacetatfällung und der Oxinfällung alle Spaltprodukte bei den Alkalien in Lösung bleiben, vor allem auch das außerordentlich starke Uran X, so daß die Operationen zur Reinigung der Alkalien besonders sorgfältig durchzuführen sind.

\section{Die Erdalkalimetalle Strontium und Barium}

Die zunächst am einfachsten erscheinende Methode der Fällung als Sulfate aus saurer Lösung kann nicht empfohlen werden, da einmal Strontiumsulfat aus mineralsauren, verhältnismäßig konzentrierten Uranylsalzlösungen nur sehr unvollständig ausfällt und da außerdem die Erdalkalisulfate, speziell Bariumsulfat, durch ein hohes Adsorptionsvermögen gekennzeichnet sind, so daß ein Aufschluß und eine nachfolgende 
gründliche Reinigung der Niederschläge unerläßlich sind. Aus diesen Gründen wurde zur Abscheidung die Fällung eines wasserlöslichen Salzes durch gleichionigen Zusatz benutzt. Sie erfolgt in stark mineralsaurer Lösung, ist grobkristallin und frei von Mischkristallbildung mit gruppenfremden Elementen und auch praktisch frei von adsorptiven Verunreinigungen.

a) Zur Fällung des Strontiums versetzt man die wässerige Phase, die man nach dem Schütteln mit Äther aus dem Scheidetrichter in ein Becherglas abzieht, mit einer Lösung von $100-200 \mathrm{mg}$ Strontiumnitrat in möglichst wenig Wasser und fällt mit 65-proz. Salpetersäure. Die Operation soll unter dem Abzug ausgeführt werden. Da die wässerige Phase niemals ganz frei von Äther ist, können ohne Kühlung unter heftiger Zersetzung. leicht Verpuffungen auftreten, die die Flüssigkeit umherschleudern und die Umstehenden ernstlich gefährden.

Die zunächst am einfachsten erscheinende Fällung des Strontiums mit Salpetersäure aus einer konzentrierten wässerigen Uranylnitratlösung ist aus mehreren Gründen nicht zu empfehlen. Die sehr stark salpetersauren Lösungen filtrieren nur sehr langsam und die bei ihrer Herstellung sich zwangsläufig ergebenden großen Volumina würden ein schnelles Arbeiten, also die Abtrennung kurzlebiger Strontium-Isotope, ausschließen. Außerdem ist aber auch Uranylnitrat in konz. Salpetersäure verhältnismäßig schwer löslich und der Niederschlag an Strontiumnitrat würde meist durch ein Vielfaches seines Gewichts an Uranylnitrat verunreinigt werden, so daß eine oder gar mehrere Umfällungen unerläßlich wären. Das Strontiumnitrat wird bei Einstellung eines Salpetersäuregehaltes entsprechend einer Säuredichte 1,455 praktisch quantitativ gefällt, doch arbeitet man bequemer mit 65-proz. Salpetersäure (d 1,40) als Fällungsmittel. Der Niederschlag ist grobkristallin, läßt sich über gehärteten oder Membranfiltern absaugen, mit 65-proz. Salpetersäure waschen und wird in möglichst wenig Wasser gelöst und erneut gefällt. Da das Strontium bei dieser Fällung als Strontiumnitrat ausfällt, bildet es mit Bariumnitrat Mischkristalle, muß also noch von Barium getrennt werden, unabhängig davon, ob Barium in wägbarer Menge zugegen war oder nicht. Zu diesem Zweck wird das Nitrat in Wasser gelöst und mit einigen mg Barium versetzt. Zur siedenden Lösung, deren Volumen mindestens
$150 \mathrm{ccm}$ betragen soll, um eine Verunreinigung des Bariums durch Strontium zu verhindern, fügt man einen großen Überschuß $(3-5 \mathrm{ccm})$ einer 10-proz. Ammonchromat- oder Kaliumbichromatlösung hinzu. Die am Filter oder Strontiumnitratniederschlag anhaftende Salpetersäure würde eine vollständige Fällung verhindern, weshalb man noch 5 ccm einer 33-proz. Ammoniumacetatlösung zur Barium-Strontium-Lösung setzt. Auf diese Weise wird die Fällung in schwach essigsaurer Lösung vollzogen und das Barium scheidet sich quantitativ ab, während Strontium in Lösung bleibt. Der Niederschlag wird auf Membranfilter filtriert und mit schwach chromathaltigem Wasser gewáschen.

Anschließend entfernt man die aus dem Strontium und Barium inzwischen entstandenen Erden Yttrium bzw. Lanthan und Cer durch eine Ammoniakfällung mit Ferrihydroxyd als Träger und scheidet aus dem Filtrat das Strontium durch Fällung mit Ammoncarbonat als Strontiumcarbonat ab. Die Fällung soll in der Wärme erfolgen.

b) Die Abscheidung des Bariums ist wesentlich einfacher, da sie über das Chlorid erfolgt, das mit dem Strontium keine Mischkristalle bildet, da das Bariumchlorid mit 2, das Strontiumchlorid mit 6 Mol. Wasser kristallisiert. Man geht also vorteilhaft vom bestrahlten Ammondiuranat aus, löst in 25-proz. Salzsäure, versetzt mit 100-200 mg Bariumchlorid in möglichst wenig Wasser und fällt mit 37-proz. Salzsäure. Der Niederschlag wird auf gehärteten Filtern abgesaugt, mit 25-proz. Salzsäure gewaschen und einmal umgefällt. Durch Waschen mit Alkohol wird zum Schluß die anhaftende Salzsäure verdrängt. Auch Millionen Teilchen etwa von Uran $\mathrm{X}$ werden bei einmaliger Umfällung des Bariumchlorids absolut quantitativ vom Barium abgetrennt.

Hat man aus besonderen Gründen Uranylnitrat bestrahlt, kann man nach der Anreicherung durch Schütteln mit Äther die wäßrige Phase mit Bariumchlorid versetzen und mit 37-proz. Salzsäure fällen. Wegen der großen Konzentration an Nitrationen besteht aber die Gefahr, daß der erste Niederschlag neben Bariumchlorid auch noch Bariumnitrat und damit also auch Strontium enthält. In diesem Falle ist es dann aus Sicherheitsgründen zu empfehlen, die Umfällung zweimal auszuführen, wodurch die Fällung bestimmt nitratfrei wird.

Die Abtrennungen wurden bisher beim Strontium in $6-8$, beim Barium in 2-4 Min. durchgeführt. 